\title{
CLIMA Y CONCENTRACIONES DE POLEN DE GRAMÍNEAS EN EL PAÍS VASCO
}

\author{
Domingo Fernando Rasilla Álvarez \\ Departamento de Geografía, Urbanismo y OT, Universidad de Cantabria, \\ Avenida de Los Castros, s/n, 39005 Santander \\ domingo.rasilla@unican.es
}

Resumen: La información sobre los niveles de calidad del aire en las ciudades españolas raramente incluye las partículas de origen orgánico, de las que el polen es un componente fundamental, responsable de numerosas patologías respiratorias y un buen marcador de los cambios ambientales. Este trabajo analiza la relación entre el polen de gramíneas medido en las tres capitales vascas y las condiciones atmosféricas, tanto a escala local como regional. Los resultados sugieren que una parte sustancial del polen de gramíneas medido en el País Vasco procede de ámbitos ajenos a esa Comunidad Autónoma, arrastrado por vientos de componente S y E y situaciones sinópticas del SW y SSE.

Palabras clave: Polen, gramíneas, clima, dinámica atmosférica.

\section{Climate and grass pollen concentrations in the Basque Country}

\begin{abstract}
Information regarding air quality in Spanish cities rarely incorporates organic particles, from which pollen is a fundamental component, responsible for numerous respiratory pathologies and a good marker of environmental changes. This contribution examines the links between grass pollen measured at the three Basque capitals and atmospheric conditions, both locally and regionally. The results suggest that a substantial part of the grass pollen measured at the Basque Country comes from nearby areas outside that Autonomous Community, pushed by easterly and southerly winds and SW and SSE circulation types.
\end{abstract}

Keywords: Pollen, Grass, climate, atmospheric dynamics. 


\section{Introducción}

La evaluación de la calidad del aire en entornos urbanos es una línea de investigación usual en el contexto de los estudios ambientales, sobre todo en lo que hace referencia a los contaminantes inorgánicos $\left(\mathrm{NO}_{2}, \mathrm{O}_{3}, \mathrm{PM}_{10}\right.$, etc...). Menos habitual son los referidos al papel del polen y otros microorganismos (esporas, virus o bacterias) en los niveles de calidad del aire, pese a que alrededor del $25 \%$ de las partículas sólidas son de origen biológico (D’Amato, 2002). Se atribuye éstas diversas patologías, como asma bronquial (DellaValle et al., 2012; Erbas et al., 2012), rinitis alérgica (Breton et al., 2006; Greiner et al., 2011) o eccemas (Eyerich et al., 2008; Werfel et al., 2015), particularmente entre niños y adolescentes. Su severidad difiere en función de condicionantes climáticos y geográficos (Damialis et al., 2019b), aunque está comprobado su agravamiento en combinación con altos niveles de contaminantes inorgánicos (Pérez-Badia et al., 2011; Todkill et al., 2020). Recientemente se ha llamado la atención acerca de la coincidencia entre el aumento de la concentración de polen en la atmósfera y de alergias en los países desarrollados; se estima que más del $20 \%$ de la población española puede sufrir algún tipo de alergia, en su mayoría por sensibilidad al polen (Baeza Ocariz, 2002; García González et al., 1999).

El polen es también un buen marcador de los cambios ambientales, tanto pasados como futuros, éstos últimos vinculados al Cambio Global, como por ejemplo transformaciones de usos de suelo o modificaciones en las condiciones atmosféricas a escala local y regional. El aumento de las concentraciones atmosféricas de gases invernadero, en particular de $\mathrm{CO}_{2}$, puede favorecer el crecimiento de las plantas, que aumentará la producción de polen e incluso, favorecer la migración de plantas alergénicas (D’Amato et al., 2007; Albertine et al., 2014; Frei and Gassner, 2008). Aunque la influencia de la temperatura sobre la cantidad de polen liberado a la atmósfera y a la duración del periodo de polinización es compleja, en función de las especies y de los climas (D'Amato and Cecchi, 2008; Weber, 2003), el calentamiento global causará una floración más temprana (Frenguelli et al., 2014). Igualmente, cambios en la cuantía y distribución estacional de las precipitaciones también incidirán en el volumen de polen en la atmósfera, ora reduciendo sus concentraciones durante la estación de polinización, ora favoreciendo el crecimiento de las plantas y el aumento de la producción de polen en los meses previos (Ribeiro et al., 2003; Tormo-Molina et al., 2010).

Estos aspectos, así como la gran incidencia de las alergias y el coste económico y social que conlleva su tratamiento, hace que los trabajos acerca de la monitorización del contenido de partículas bióticas y la identificación de los mecanismos de producción y liberación de compuestos alergénicos sea de gran interés para las autoridades de salud ambiental y de la población en general, anticipando la intensidad y duración de los periodos de polinización, planificación de actividades sin riesgo a ataques de alergias etc... 
El objetivo de este trabajo es el análisis de la relación entre el clima y la concentración de gramíneas (Poaceae) en las tres capitales vascas. Las gramíneas, el grupo de plantas más abundantes (unos 700 géneros y 12.000 especies), suponen el $20 \%$ de la vida sobre el planeta y dominan los paisajes de latitudes templadas, tanto de forma espontánea como cultivada; como tal, son la fuente de alimentación directa no sólo de los herbívoros, sino también de humanos (cereales). Constituyen uno de los pólenes con mayor capacidad alérgica en España, particularmente en las regiones del norte, donde se aprecian los porcentajes de sensibilización de hasta un 85\% en Vitoria o el 80\% en Bilbao (Benito y Soto, 2001; Subiza, 2003). Para conseguir ese objetivo, este trabajo:

- Realiza un caracterización espacial y temporal del polen de gramíneas en el País Vasco.

- Determina las condiciones atmosféricas a escala local y regional favorables a episodios extremos de concentración de polen de gramíneas.

\section{Materiales y Métodos}

La información polínica procede de la Red para el control de Polen de la Comunidad Autónoma Vasca, perteneciente al Laboratorio de Salud Pública, obtenidos a través de la página web del Gobierno Vasco (https://opendata.euskadi.eus/). El periodo de análisis abarca desde el año 2014 hasta el año 2020. En el muestreo del polen se emplea el método de succión de Hirst, y las muestras diarias, recogidas en los captadores Burkard, son preparadas para su lectura en el microscopio óptico, controlándose un total de cuarenta y cinco tipos polínicos diferentes y las esporas de Alternaria. Las concentraciones diarias aparecen expresadas como número de granos de polen por metro cúbico de aire $\left(\mathrm{g} / \mathrm{m}^{3}\right.$; Aguinagalde et al., 2020).

A partir de esa información polínica se definieron los siguientes parámetros para cada una de las ciudades:

1. Fechas de comienzo, de fin y duración de la estación de polinización, determinada siguiendo el método de los porcentajes. Este es uno de los más comunes, basado en la eliminación de un cierto porcentaje de días al comienzo y final del año (Andersen, 1991). Se ha utilizado el porcentaje del $90 \%$, los que implica que el comienzo de la estación polínica ocurrirá en el día en el que se haya acumulado el $5 \%$ del total de polen correspondiente a ese año, y el final corresponderá al día en el que se haya acumulado el $95 \%$.

2. Suma anual de polen e integral polínica.

3. Número de días con concentraciones extremas de polen: se ha optado por un criterio "climatológico" en vez de uno "alergológico", considerando aquellos en los que la concentración de polen de gramíneas fue igual o superior al percentil 95 en cada localidad. 
Todos los cálculos fueron realizados con el paquete de R AeRobiolgy (Rojo et al., 2019).

La información de carácter meteorológico proviene de estaciones sinópticas de AEMET localizadas en las proximidades de las tres ciudades (Tabla 1), obtenida de las páginas web del NOAA (Integrated Surface Database; ftp://ftp.ncei.noaa.gov/pub/data/noaa/) y del European Climate Assessment \& Dataset (https://www.ecad.eu/dailydata/index.php). Los parámetros meteorológicos seleccionados fueron la temperatura (máxima y mínima), la humedad relativa media diaria, la dirección y velocidad del viento (a las 12 UTC), el porcentaje de insolación diaria, la radiación solar (media diaria), la nubosidad media diaria y la lluvia acumulada en 24 horas. A partir de los datos en bruto se calcularon, para la estación de polinización y durante los dos meses previos, los siguientes índices climáticos:

1. Promedio de la temperatura (máxima y mínima), de la nubosidad y de la insolación, precipitación acumulada y número de días de precipitación (según los siguientes umbrales: > $1 \mathrm{~mm}, 1$ a $10 \mathrm{~mm}, 10-30 \mathrm{~mm}$ y $>30 \mathrm{~mm}$ ).

2. Grados día de crecimiento, calculados según la siguiente fórmula:

$$
G D D=\frac{T_{\max +} T_{\min }}{2}-T_{\text {base }}
$$

Siendo $\mathrm{T}_{\max }$ la temperatura máxima diaria, $\mathrm{T}_{\min }$ la temperatura mínima diaria y $\mathrm{T}_{\text {base }}$ la temperatura base. Los grados día de crecimiento hasta el comienzo de la estación de polinización se calculan acumulando el número de grados día desde el 1 de enero. Se consideró como $\mathrm{T}_{\text {base }} 5^{\circ} \mathrm{C}$, considerada el umbral de temperatura de crecimiento en especies de clima templado (Dahl et al., 2013), siempre que la temperatura máxima diaria sea superior a la temperatura base; en caso contrario, se considera $0^{\circ} \mathrm{C}$.

Tabla 1. Localización de las estaciones meteorológicas

\begin{tabular}{|l|l|c|c|c|}
\hline Código OMM & Nombre & Latitud & Longitud & Altitud \\
\hline 08025 & Bilbao-Sondica & 43,301 & $-2,911$ & 42 \\
\hline 08207 & San Sebastián- Igueldo & 43.300 & $-2,033$ & 259 \\
\hline 08080 & Vitoria-Foronda & 42.883 & $-2,724$ & 512 \\
\hline
\end{tabular}

Esos índices climáticos fueron puestos en relación con los índices aerobiológicos anteriormente citados, cuantificándose mediante el cálculo del coeficiente de correlación de Spearman. La elección de este coeficiente de correlación es su mayor robustez y resistencia a datos extremos en series de corta longitud (tan sólo 7 elementos), frente al más convencional coeficiente de Pearson (Wilks, 2011). 
Para comprender mejor los mecanismos de transporte del polen por el viento e inferir sus posibles orígenes geográficos se han utilizado los diagramas polares, que representan simultáneamente las concentraciones de polen según la dirección y velocidad del viento en cada localidad, incluido dentro del paquete de R "OpenAir" (https:// davidcarslaw.github.io/openair/).

Si bien las relaciones entre la dinámica polínica local y variables meteorológicas están bien fundamentadas en la literatura científica, la fisiología de las plantas está condicionada por una combinación de condiciones atmosféricas. La agrupación de las posibles combinaciones en un número reducido de categorías es una práctica común para simplificar las complejas interrelaciones entre las variables meteorológicas y clarificar sus vínculos con la dinámica de las plantas (Laiidi 2001; Makra et al. 2006; Hebbern y Cakmak 2015; Dixon et al., 2016). La clasificación de tipos de circulación de Lamb ha sido elegida por su fácil elaboración y sencillez, dado que describe la configuración del mapa de presión a nivel del mar, a partir del cálculo de índices atmosféricos (dirección y velocidad del viento geostrófico y vorticidad), agrupados posteriormente en categorías (tipos de circulación), dependiendo del predominio de movimientos verticales (ascendentes -tipo ciclónico, C- o descendentes -tipo anticiclónico, A-) o advectivos (en 8 categorías -N,NE, E, SE, S, SW, W, NW- según la rosa de los vientos), más un tipo indeterminado (-U-). En este estudio, se ha utilizado una variante simplificada de la clasificación original, que reduce los 26 tipos originales a sólo 8, rotando ligeramente los límites entre los tipos advectivos y reduciendo el número de categorías (Fernández y Rasilla, 2001). El posible nexo entre la circulación a escala sinóptica y las concentraciones de polen se evaluó aplicando el índice PI (Performance Index; Paschalidou et al., 2020):

$$
P I=\left(\frac{\left(\frac{\text { Concentración total de polen en el tipo sinóptico }_{i}}{\text { Concentración total de polen }}\right)}{\left(\frac{\text { Frecuencia }(\text { número de días) del tipo sinóptico }}{i}\right)}-1\right) * 100
$$

Valores próximos a 0 indican que los niveles de polen se distribuyen de manera equitativa entre todos los tipos sinópticos, mientras que valores positivos (negativos) denotan que niveles elevados de polen son más (menos) frecuentes en esas categorías; valores próximos a -100 identifican categorías sin polen.

Por último, también se han utilizado retro-trayectorias de las partículas de aire que, a diversas alturas sobre la superficie (500, 1500 y 3000 m) alcanzaron el País Vasco en días seleccionados con alta carga polínica de gramíneas (https://www.ready.noaa.gov/ HYSPLIT.php). El lapso de tiempo seleccionado fue 72 horas previas al día de interés. 


\section{Resultados}

\subsection{Caracterización espacio-temporal de la concentración de gramíneas en el País Vasco}

A partir de los datos de polen obtenidos en el periodo 2014-2020, los niveles de carga polínica en las principales ciudades del País Vasco son los típicos de un clima oceánico, más reducidos que los de la España Mediterránea (Tabla 2; Subiza et al., 2003). La ciudad con más carga polínica es Vitoria, mientras que San Sebastián recibe aproximadamente el $75 \%$ de la anterior. Los táxones más frecuentes son los de Pinus (23\% en Bilbao, pero sólo 7,1\% en Vitoria), Cupressacea/Taxacea (entre el 8,6 \% en San Sebastián y el 17,8 \% en Vitoria), Quercus (entre el 11,2 y el 16,5\%) y Urticaceae (entre el 8 y el $13,5 \%)$.

Tabla 2. Niveles de carga polínica en el País Vasco

\begin{tabular}{|l|c|c|}
\hline Localidad & Valor medio anual $\left(\right.$ granos $\left./ \mathrm{m}^{3}\right)$ & \% de polen de gramineas \\
\hline Bilbao & 23933,1 & 8,7 \\
\hline San Sebastián & 18485,8 & 7,5 \\
\hline Vitoria & 25512,4 & 9,7 \\
\hline
\end{tabular}

Las gramíneas, pese a su elevado potencial alergénico, constituyen menos del $10 \%$ del polen medido, mostrando una menor variabilidad espacial que la de otros táxones, y un claro ciclo anual. La estación polínica de las gramíneas se inicia de manera general a fines de abril y comienzos de mayo, y se prolonga hasta el mes de agosto, aunque su finalización no es simultánea (Figura 1). La duración total muestra, por tanto, una acusada variabilidad espacial. Obsérvese que la duración de la estación polínica no guarda relación con la cantidad total de polen recogida: Vitoria es la ciudad que más polen de gramíneas recoge, pero la duración de la estación polínica no llega a los 100 días, frente a más de 140 en las restantes. Los máximos diarios suelen aparecer entre la segunda quincena de mayo (Bilbao) y la primera de junio (Vitoria).

Tampoco se aprecia demasiada coherencia espacial, con Vitoria y San Sebastián mostrando una correlación interanual elevada (coeficiente de correlación $r$ de 0,89, significativa a un nivel de confianza del $95 \%)$ frente a Bilbao $(0,53$, no significativa al nivel de confianza del 95\%). Por ello es difícil hablar de un comportamiento común para todas las localidades: sólo 2017 registró los valores mínimos en todas las localidades, mientras que la alta producción de 2020 se centró en Vitoria y San Sebastián. 


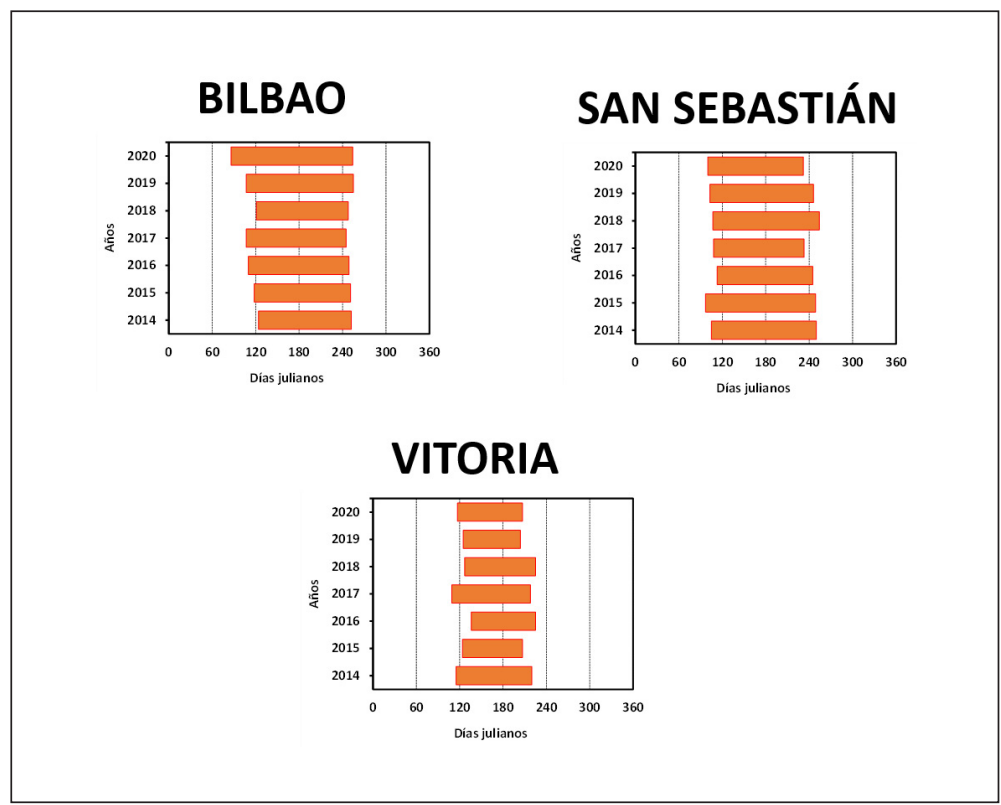

Figura 1. Duración de la estación polínica de gramíneas.

Fuente: Gobierno Vasco (https://opendata.euskadi.eus/). Elaboración propia.

\subsection{Caracterización de las condiciones atmosféricas locales y regionales favorables a episodios extremos de concentración de gramíneas}

La presencia de polen en la atmósfera responde a una serie de procesos que intervienen en su formación, liberación, transporte y deposición, agrupados bajo la denominación genérica de "procesos aerobiológicos". Estos actúan a dos escalas temporales: por un lado, el calor acumulado es fundamental para el inicio de la floración; por otro lado, una vez liberado el polen a la atmósfera (polinización anemófila), ésta participa como medio de dispersión (un mecanismo energéticamente poco costoso aumenta la tasa de reproducción) y deposición, aunque el tiempo de permanencia está inversamente relacionado con el tamaño del polen. En esta fase, las variables que afectan de forma más directa son otra vez la temperatura, la precipitación y el viento, y en menor medida, la humedad relativa y la insolación. En consecuencia, se ha procedido a un análisis del papel de la variabilidad atmosférica en relación con la fecha de inicio de la estación polínica, que marca el inicio de la floración, y, por otro lado, en relación con el volumen de la producción polínica y la duración de este periodo.

A partir de los datos de la Tabla 3, las fechas de inicio de la estación polínica sólo están correlacionadas de manera evidente con los valores meteorológicos de los me- 
ses de marzo y abril en Álava. Aquí se registran correlaciones negativas entre dicha fecha y la temperatura máxima, el número de grados día y el porcentaje de insolación relativa; las únicas correlaciones positivas aparecen en el caso del número de días de precipitación y la nubosidad. De acuerdo con estos valores, condiciones frescas y húmedas se traducen en un retraso de la llegada de la primavera.

Tabla 3. Valor del coeficiente de correlación de Spearman entre fechas de inicio (días julianos) de la estación polínica de gramíneas y diferentes variables meteorológicas en el País Vasco durante los dos meses previos (marzo y abril). Valores superiores (inferiores) a $\pm 0,7$ son significativos al nivel de confianza del 95 \% según la prueba de Student

\begin{tabular}{|l|c|c|c|c|c|c|c|c|}
\hline & $\begin{array}{c}\text { Tmáx } \\
{ }^{o} C\end{array}$ & $\begin{array}{c}\text { Tmín } \\
{ }^{o} C\end{array}$ & $\begin{array}{c}G D D \\
{ }^{\circ} C\end{array}$ & $\begin{array}{c}H r \\
\%\end{array}$ & $\begin{array}{c}\text { PP } \\
M m\end{array}$ & $\begin{array}{c}\text { Días } \\
\text { PP }\end{array}$ & $\begin{array}{c}\text { Ins } \\
\%\end{array}$ & $\begin{array}{c}\text { Nub } \\
\text { Oktas }\end{array}$ \\
\hline Bilbao & $-0,08$ & 0,11 & 0,01 & $-0,51$ & 0,31 & 0,36 & 0,12 & $-0,14$ \\
\hline San Sebastián & $-0,45$ & $-0,59$ & $-0,55$ & $-0,41$ & 0,04 & 0,15 & 0,02 & 0,02 \\
\hline Vitoria & $-0,96$ & 0,01 & $-0,82$ & 0,37 & 0,46 & 0,78 & $-0,79$ & 0,71 \\
\hline
\end{tabular}

Fuente: Gobierno Vasco (https://opendata.euskadi.eus/), NOAA (ftp://ftp.ncei.noaa.gov/pub/data/noaa/) y ECAD (https://www.ecad.eu/). Elaboración propia.

También se correlacionó la duración de la estación polínica y la integral correspondiente a dicha estación con las variables meteorológicas correspondientes al periodo mayo-agosto. Los resultados ponen de manifiesto la ausencia de relaciones claras en ambos casos, con comportamientos opuestos en el caso de Bilbao y Vitoria para la duración (insolación y nubosidad). Para la integral polínica son destacables la importancia de la insolación/nubosidad, acompañada de la temperatura mínima y los grados día en Bilbao.

Tabla 4. Valor del coeficiente de correlación de Spearman entre la duración (días) de la estación polínica de gramíneas y diferentes variables meteorológicas en el País Vasco

(desde mayo a agosto). Valores superiores (inferiores) a \pm 0.71 son significativos al nivel de confianza del 95 \% según la prueba de Student

\begin{tabular}{|l|c|c|c|c|c|c|c|c|}
\hline & $\begin{array}{c}\text { Tmáx } \\
{ }^{o} C\end{array}$ & $\begin{array}{c}\text { Tmín } \\
{ }^{o} C\end{array}$ & $\begin{array}{c}G D D \\
{ }^{o} C\end{array}$ & $\begin{array}{c}H r \\
\%\end{array}$ & $\begin{array}{c}\text { PP } \\
\text { Mm }\end{array}$ & $\begin{array}{c}\text { Dias } \\
\text { PP }\end{array}$ & $\begin{array}{c}\text { Ins } \\
\%\end{array}$ & $\begin{array}{c}\text { Nub } \\
\text { Oktas }\end{array}$ \\
\hline Bilbao & 0,39 & 0,03 & 0,24 & $-0,30$ & 0,25 & 0,21 & 0,90 & $-0,67$ \\
\hline San Sebastián & $-0,50$ & $-0,33$ & $-4,48$ & 0,71 & 0,18 & 0,29 & $-0,08$ & 0,19 \\
\hline Vitoria & $-0,31$ & 0,17 & $-0,12$ & 0,34 & 0,36 & 0,23 & $-0,76$ & 0,79 \\
\hline
\end{tabular}

Fuente: Gobierno Vasco (https://opendata.euskadi.eus/), NOAA (ftp://ftp.ncei.noaa.gov/pub/data/noaa/) y ECAD (https://www.ecad.eu/). Elaboración propia. 
Tabla 5. Valor del coeficiente de correlación de Spearman entre la integral (suma total de granos de polen) de la estación polínica de gramíneas y diferentes variables meteorológicas en el País Vasco (desde mayo a agosto). Valores superiores (inferiores) a \pm 0.71 son significativos al nivel de confianza del 95 \% según la prueba de Student

\begin{tabular}{|l|c|c|c|c|c|c|c|c|}
\hline & $\begin{array}{c}\text { Tmáx } \\
{ }^{o} C\end{array}$ & $\begin{array}{c}\text { Tmín } \\
{ }^{o} C\end{array}$ & $\begin{array}{c}G D D \\
{ }^{o} C\end{array}$ & $\begin{array}{c}H r \\
\%\end{array}$ & $\begin{array}{c}\text { PP } \\
M m\end{array}$ & $\begin{array}{c}\text { Dias } \\
P P\end{array}$ & $\begin{array}{c}\text { Ins } \\
\%\end{array}$ & $\begin{array}{c}\text { Nub } \\
\text { Oktas }\end{array}$ \\
\hline Bilbao & $-0,53$ & $-0,87$ & $-0,79$ & $-0,62$ & $-0,46$ & $-0,25$ & 0,52 & $-0,64$ \\
\hline San Sebastián & 0,09 & $-0,13$ & 0,00 & $-0,02$ & 0,39 & 0,34 & 0,90 & $-0,77$ \\
\hline Vitoria & 0,33 & $-0,13$ & 0,15 & $-0,31$ & 0,07 & 0,25 & 0,58 & $-0,85$ \\
\hline
\end{tabular}

Fuente: Gobierno Vasco (https://opendata.euskadi.eus/), NOAA (ftp://ftp.ncei.noaa.gov/pub/data/noaa/) y ECAD (https://www.ecad.eu/). Elaboración propia.

La ausencia de correlaciones estrechas a escala estacional condujo a un análisis exploratorio a escala diaria, para determinar posibles relaciones ocultas con el uso de promedios a largo plazo. Para ello, los días incluidos dentro del periodo considerado como estación de polinización en cada localidad fueron clasificados en dos categorías, de acuerdo con el contenido de polen, y comparados entre sí. Una de las categorías fue la de días extremos, es decir, con una concentración de polen superior al percentil 95. Las variables meteorológicas comparados fueron las anomalías de la temperatura máxima, el \% de insolación, la humedad relativa, la nubosidad y el número de días de precipitación según categorías. Los resultados aparecen en la Figura 2.

En este caso, los resultados apuntan a relaciones más consistentes entre carga polínica y variables meteorológicas. En el caso del País Vasco, los días con alta carga polínica de gramíneas son cálidos y con una baja humedad relativa, cielos poco nubosos y alta insolación. Obsérvese, por último, que todas esas condiciones favorables no son propicias a precipitaciones abundantes, puesto que el lavado de las capas bajas por la lluvia conduce a la rápida deposición del polen. No obstante, algunos eventos significativos están acompañados de precipitaciones moderadas, como consecuencia de la evolución de la dinámica atmosférica que revisaremos posteriormente.

En relación al papel del viento y el polen, es posible diferenciar dos tipos de transporte: a escala local, en el que las partículas son depositadas en las proximidades de la fuente de producción, y a escala regional, donde la circulación sinóptica propicia el desplazamiento a localidades alejadas de su origen. Las relaciones entre ambos son complejas: vientos fuertes diluyen el contenido polínico, mientras que velocidades reducidas no agitan las anteras e impiden la liberación de polen; en otros casos, provoca la re-suspensión del polen ya sedimentado, lo que explicaría la aparición de tipos polínicos en una localidad fuera de su periodo de polinización. Para solventar estas dificultades, se ha recurrido al uso de gráficas polares, que representan las diferentes concentraciones de po- 
a)

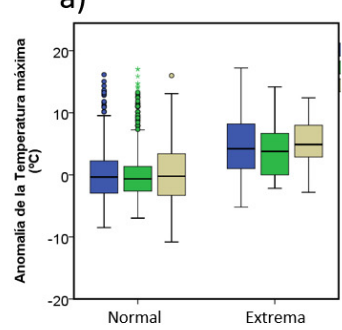

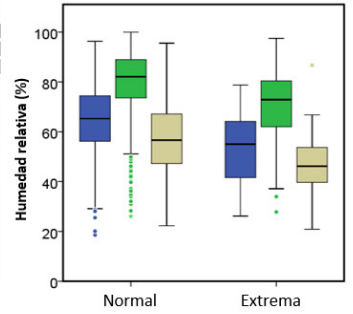

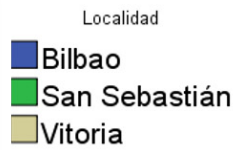

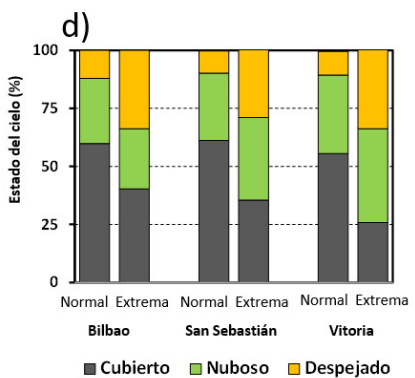

e)

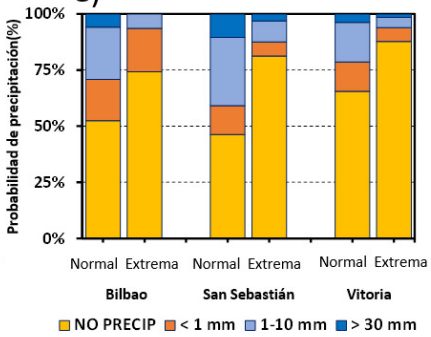

Figura 2. Caracterización de las condiciones atmosférica locales durante los días de concentración de polen de gramíneas normal y extrema en las localidades de referencia.

a) Anomalía de la temperatura máxima $\left.\left({ }^{\circ} \mathrm{C}\right) ; \mathrm{b}\right)$ anomalía de la radiación solar $\left(\mathrm{w} / \mathrm{m}^{2}\right)$;

c) humedad relativa media (\%); d) probabilidad de estado del cielo (octas);

e) probabilidad de la precipitación según intervalos ( $\mathrm{mm} / \mathrm{día})$.

Fuente: NOAA (ftp://ftp.ncei.noaa.gov/pub/data/noaa/) y ECAD (https://www.ecad.eu/). Elaboración propia.

len según la dirección y velocidad del viento, lo que facilita una primera identificación de las fuentes potenciales de polen y su influencia respecto a los niveles globales en las estaciones. Su análisis para cada una de las capitales vascas ofrece interesantes aspectos. En el caso de las concentraciones medias (Figura 4, izquierda), destaca el predominio de concentraciones elevadas con vientos relativamente suaves (velocidades inferiores a 4-6 $\mathrm{m} / \mathrm{s}$ ) y de componente S y NE, en clara adaptación a la topografía local (Figura 3). Estos vientos no son los más frecuentes en la región durante la estación de polinización, ya que predominan los de componente W y NW (vinculados a las situaciones inestables atlánticas) y las brisas locales (NNW en Bilbao, N en San Sebastián, NE en Vitoria). De acuerdo con las gráficas, éstas últimas componentes son las más limpias, particularmente en San Sebastián. Sin embargo, en el caso de los días de máxima concentración de contaminantes (Figura 3, derecha), los vientos de componente S desaparecen (salvo en Bilbao), mientras que permanecen los de componente E. 


\section{BILBAO}

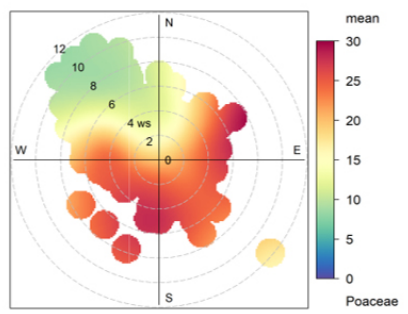

SAN SEBASTIÁN

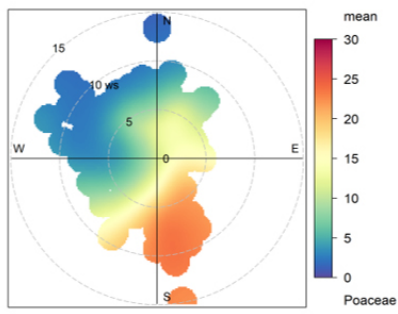

SAN SEBASTIÁN
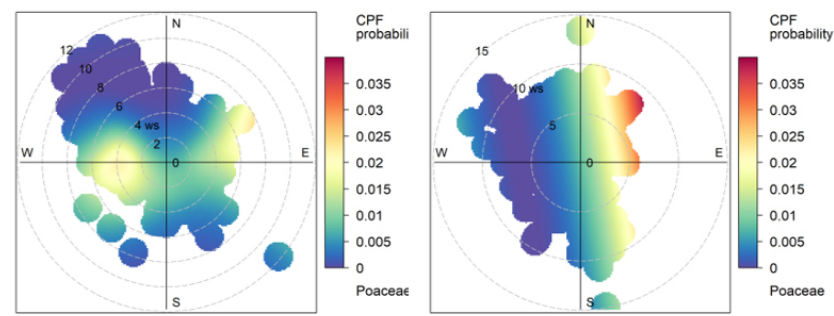

Figura 3. Rosas polínicas.

\section{VITORIA}

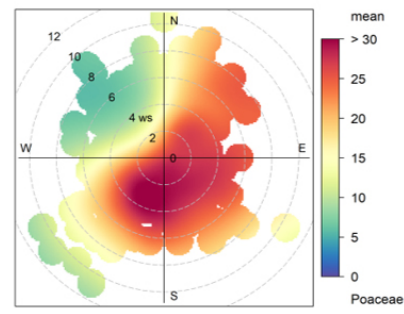

VITORIA

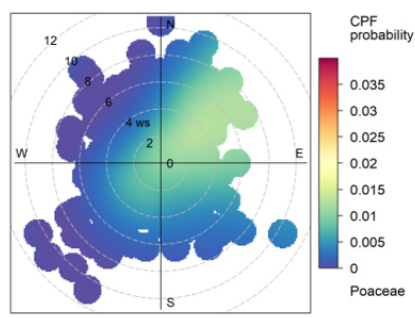

Fuente: Gobierno Vasco (https://opendata.euskadi.eus/) y NOAA (ftp://ftp.ncei.noaa.gov/pub/data/noaa/).

Elaboración propia.

Las circulaciones locales del S y E tienen su correspondencia con el predominio de elevadas concentraciones de polen bajo situaciones sinópticas del SSE y del SW, y en menor medida, ciclónicas, en correspondencia con jornadas cálidas, secas y luminosas en toda la región (Figura 4). Por el contrario, las circulaciones más limpias son, de nuevo, las que propician la llegada de masas de aire fresco y húmedo desde el Atlántico (NNW y NNE), normalmente acompañadas de precipitaciones abundantes que "lavan" la atmósfera. Obsérvese, además, que los valores más elevados del índice PI en Bilbao y San Sebastián respecto a Vitoria confirman un fondo más elevado en esta última localidad; el impacto de las citadas situaciones atmosféricas sobre los niveles de polen es muy superior en las localidades costeras con respecto a sus niveles de fondo habituales.

De acuerdo con la información proporcionada por las rosas de contaminación (figura 3) y por el catálogo de tipos de circulación (figura 4), los días con niveles de polen de gramíneas elevados corresponden a la llegada de masas de aire procedentes del $\mathrm{S}$, y ocasionalmente del E. Un procedimiento adicional para confirmar ambas hipótesis es el cálculo de retrotrayectorias, que permiten conocer el recorrido de una partícula (polén) días antes de alcanzar un punto (Figura 5). El día 25 de mayo de 2017 pertenece a la categoría ciclónica, debido a la expansión de la baja térmica africana hacia el N; un 


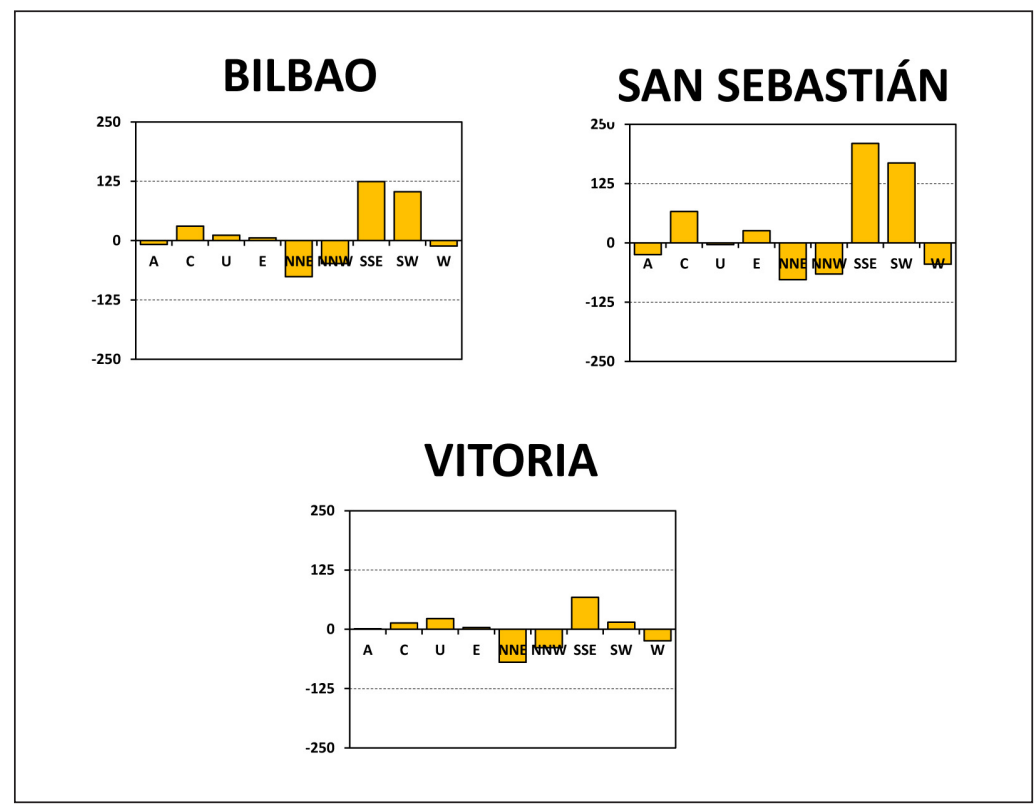

Figura 4. Valores de PI correspondientes a la concentración de gramíneas según tipos de circulación.

Fuente: Gobierno Vasco (https://opendata.euskadi.eus/). Elaboración propia. Elaboración propia.

anticiclón se sitúo sobre Europa Occidental. Este dispositivo facilitó la recirculación del aire sobre la Península Ibérica, alcanzando el País Vasco tras recorrer el valle del Ebro y el sector oriental de la Meseta. El día 27 de mayo de 2020 el anticiclón de bloqueo se desplazó hacia el W, situándose sobre las Islas Británicas, dirigiendo un flujo del E, que evolucionó días siguientes al SE. Las retrotrayectorias muestran la llegada de una masa de aire que ha recorrido Europa Occidental en los días precedentes.

La ausencia de correlaciones estrechas a nivel local entre concentraciones de polen y variables meteorológicas, la similitud de la circulación atmosférica y de las trayectorias deducidas a partir del análisis de algunos eventos relevantes sugiere que, al menos una parte del polen de gramíneas medido en las capitales vascas procede de fuentes lejanas, que alcanzarían el País Vasco siguiendo escenarios de transporte específicos, en los que la posición de las altas presiones sobre Europa Occidental parece determinante. Dentro de estos patrones sinópticos, la topografía local modularía los vientos en superficie, transportando polen de gramíneas desde el S de Francia, el valle del Ebro o incluso la Meseta. Esa hipótesis justificaría también la primacía de Álava en cuanto a carga polínica, cuyos niveles de base responden tanto a su mayor superficie agrícola (27\%), en gran parte dedicada a cereales (66\%) y plantas forrajeras (2\%), como a su proximidad a esas hipotéticas fuentes de gramíneas (Tabla 6). 

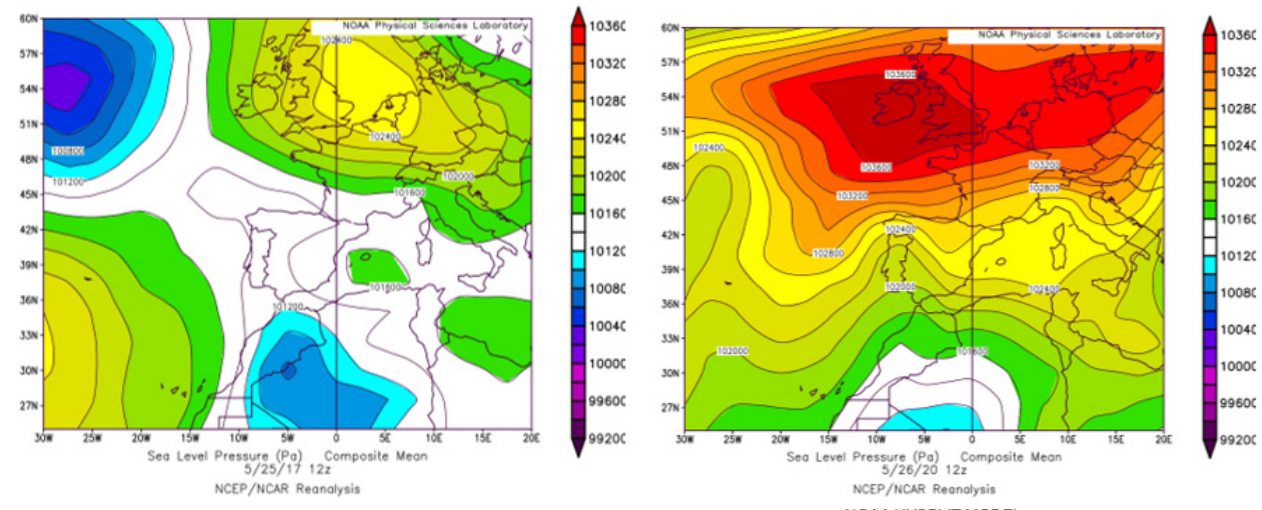

NOAA HYSPLIT MODEL

Backward trajectories ending at 1200 UTC 25 May 17 NOAA HYSPLIT MODEL

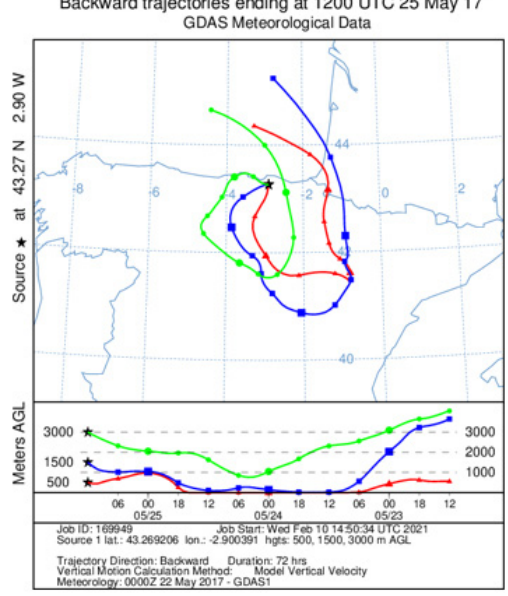
Backward trajectories ending at 1200 UTC 27 May 20 GDAS Meteorological Data

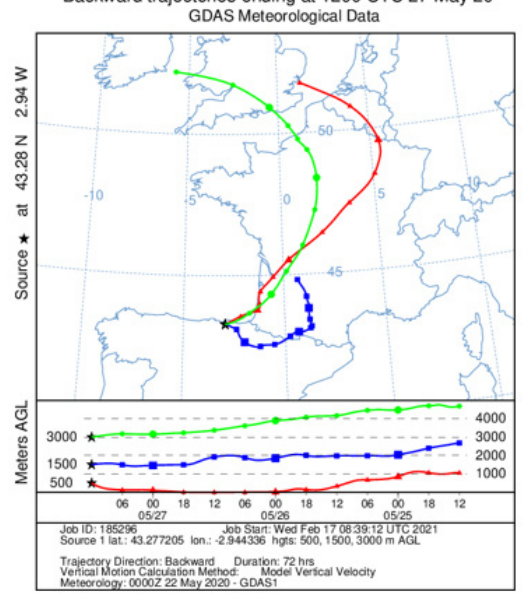

Figura 5. Mapa de la presión en superficie (conjunto superior) y retroyectorias correspondientes a los días 25 de mayo de 2017 (izquierda) y 27 de mayo de 2020.

Fuente: NOAA/ESRL Physical Sciences Laboratory (http://psl.noaa.gov/) y Air Resources Lab (https://www.ready.noaa.gov/HYSPLIT.php). Elaboración propia.

Tabla 6. Distribución (\%) de usos del territorio a nivel provincial en el País Vasco

\begin{tabular}{|l|c|c|c|c|c|c|c|}
\hline & Bosque & Matorral & Pastizal-matorral & Prados & Herbazal & Agrícola & Otros \\
\hline Álava & 47 & 6 & 7 & 5 & 2 & 27 & 7 \\
\hline Vizcaya & 60 & 5 & 4 & 16 & 1 & 1 & 13 \\
\hline Guipúzcoa & 63 & 4 & 3 & 19 & 2 & 1 & 9 \\
\hline
\end{tabular}

Fuente: Gobierno Vasco (https://www.euskadi.eus/distribucion-de-los-usos-del-suelo-de-la-c-a-de-euskadi-por-ambitos-territoriales/web01-a2garrai/es/). Elaboración propia. 


\section{Discusión y conclusiones}

La relación entre las condiciones atmosféricas y la dinámica del polen es conocida y se desarrolla a diferentes escalas espaciales y temporales. A escala local, la liberación y dispersión inicial del polen está controlada por variables como temperatura, precipitación, viento etc.; sin embargo, cuando no existen evidencias de dicha relación es posible inferir como origen de las concentraciones de polen registradas en un lugar específico el transporte desde regiones vecinas. Diferentes estudios han confirmado este transporte a larga distancia, que en algunos casos pueden superar los 2000 km (Izquierdo et al., 2011; Bilinska et al., 2017; Izquierdo et al., 2017). En relación con este aspecto, el presente trabajo, tras caracterizar espacial y temporalmente la carga polínica de gramíneas en el País Vasco, ha identificado cuáles son las condiciones atmosféricas más favorables, tanto a escala local como regional, para la recepción de elevados volúmenes de ese tipo de polen, planteando la hipótesis de un aporte sustancial procedente de regiones limítrofes.

En líneas generales, la carga polínica registrada en las capitales vascas alcanza valores típicos de los climas oceánicos, siendo Álava la que destaca por su mayor carga, aunque la duración de la estación favorable es la más reducida. Con respecto a las condiciones en los meses previos, sólo se han obtenido valores significativos en Álava, señalando una clara vinculación con la llegada de la primavera, de tal manera que primaveras frescas y húmedas la retrasan, mientras que las cálidas la adelantan. En relación con el impacto durante la estación de polinización no se ha encontrado un patrón claro de conexiones significativas.

Sin embargo, estos condicionantes no significan la ausencia total de relación. Los días con elevados valores de carga polínica de gramíneas son habitualmente cálidos, poco nubosos y mayoritariamente secos, con vientos suaves (alrededor de 4-6 m/s) de componente este, sureste y sur. En consonancia con ello, las situaciones atmosféricas características que propician elevados valores de polen son las del SSE y SSW.

\section{Bibliografía}

Aguinagalde Aizpurua, X. (2020). Polen en el aire de la CAV. Laboratorio de Salud Pública. Dirección de Salud Pública y Adicciones. Departamento de Salud. Gobierno Vasco, 45 p.

Albertine J.M., Manning W.J., DaCosta M., Stinson K.A., Muilenberg M.L., Rogers C.A. (2014). Projected carbon dioxide to increase grass pollen and allergen exposure despite higher ozone levels. PLoS One 9 (11), e111712. DOI: http://dx.doi.org/10.1371/journal.pone.0111712

Andersen T.B. (1991). A model to predict the beginning of the pollen season. Grana, 30(1), pp. 269-275. DOI: http://dx.doi.org/10.1080/00173139109427810

Antépara I., Fernández J.C., Gamboa P., Jaúregui I. y Miguel F. (1995). Pollen allergy in the Bilbao area (European Atlantic seaboard climate). pollination forecasting methods. Clinical \& Experimental Allergy, 25(2), pp. 133-140. DOI: http://dx.doi.org/10.1111/j.1365-2222.1995.tb01018.x 
Baeza M.L. (2002). Nuevos alérgenos: nuevas patologías alérgicas. Allergol Inmmunol Clin, 17(2), pp. 61-62.

De Benito V. y Soto J. (2001). Polinosis y aerobiología del polen en la atmósfera de Santander. Alergol Inmunol Clin 16: pp. 84-90.

Bilinska D., Skjøth C.A., Werner M., Kryza M., Malkiewicz, Krynicka J. y Drzeniecka-Osiadacz A. (2017). Source regions of ragweed pollen arriving in south-western Poland and the influence of meteorological data on the HYSPLIT model results. Aerobiologia 33: pp. 315-326. DOI: http://dx.doi.org/10.1007/s10453-017-9471-9

Bousquet J., Anto J.M., Annesi-Maesano I., Dedeu T., Dupas E., Pépin J.L., Eyindanga L.S.Z., Arnavielhe S., Ayache J., Basagana X., Benveniste S., Venturos N.C., Chan H.K., Cheraitia M., Dauvilliers Y., Garcia-Aymerich J., Jullian-Desayes I., Dinesh C., Laune D., Dac J.L., Nujurally I., Pau G., Picard R., Rodo X., Tamisier R., Bewick M., Billo N.E., Czarlewski W., Fonseca J., Klimek L., Pfaar O., Bourez J.M. (2018). POLLAR: Impact of air POLLution on Asthma and Rhinitis; a European Institute of Innovation and Technology Health (EIT Health) project. Clin Transl Allergy 17: pp. 8-36. DOI: http://dx.doi.org/10.1186/s13601-018-0221-z

Breton M.C., Garneau M., Fortier I., Guay F. y Louis J. (2006). Relationship between climate, pollen concentrations of Ambrosia and medical consultations for allergic rhinitis in Montreal, 1994-2002. Science of the Total Environment, 370: pp. 39-50. DOI: http://dx.doi.org/10. 1016/j.scitotenv.2006.05.022.

Caillaud D.M., Martin S., Segala C., Vidal P., Lecadet J., Pellier S., Rouzaire P., Tridon A. y Evrard B. (2014). Airborne pollen levels and drug consumption for seasonal allergic rhinoconjunctivitis: a 10 year study in France et al. Airborne pollen levels and drug consumption for seasonal allergic rhinoconjunctivitis: a 10-year study in France. Allergy 70: pp. 99-106. DOI: http:// dx.doi.org/10.1111/all.12522.

Carslaw D.C. y Ropkins K. (2012). Openair: an R package for air quality data analysis. Environmental Modelling \& Software, 27-28: pp. 52-61. DOI: http://dx.doi.org/10.1016/j.envsoft. 2011.09.008

D'Amato G. (2002). Environmental urban factors (air pollution and allergens) and the rising trends in allergic respiratory diseases. Allergy, 54 (72), pp. 30-33. DOI: http://dx.doi.org/10. 1034/j.1398-9995.57.s72.5.x

D'Amato G. y Cecchi L. (2008). Effects of climate change on environmental factors in respiratory allergic diseases. Clin. Exp. Allergy 38: pp. 1264-1274. DOI: http://dx.doi.org/ 10.1111/j.13652222.2008.03033.x

D’Amato G., Cecchi L., Bonini S., Nunes C., Annesi-Maesano I., Behrendt H., Liccardi G., Popov T. y van Cauwenberge P. (2007). Allergenic pollen and pollen allergy in Europe. Allergy 62: pp. 976-990. DOI: http://dx.doi.org/ 10.1111/j.1398-9995.2007.01393.x

Dahl A., Galán C., Hajkova L., Pauling A., Sikoparija B., Smith M. y Vokou D. (2013). The onset, course and intensity of the pollen season. In Sofiev M, Bergmann KC (eds). Springer, Netherlands, Dordrecht, pp. 29-70. DOI: http://dx.doi.org/10.1007/978-94-007-4881-1_3

Damialis A., Traidl-Hoffmann C. y Treudler R. (2019). Climate Change and Pollen Allergies. Biodiversity and Health in the Face of Climate Change. Springer, pp. 47-66. DOI: http://dx.doi.org/ 10.1007/978-3-030-02318-8_3 
DellaValle C.T., Triche E.W., Leaderer B.P. y Bell M.L. (2012). Effects of ambient pollen concentrations on frequency and severity of asthma symptoms among asthmatic children. Epidemiology 23, pp. 55-63. DOI: http://dx.doi.org/10.1097/EDE.0b013e31823b66b8

Dixon P.G., Allen M., Gosling S.N., Hondula D.M., Ingole V., Lucas R. y Vanos J. (2016). Perspectives on the synoptic climate classification and its role in interdisciplinary research. Geography Compass, 10, pp. 147-164. DOI: http://dx.doi.org/10.1111/gec3.12264

Erbas B., Akram M., Dharmage S.C., Tham R., Dennekamp M., Newbigin E., Taylor P., Tang M.L.K. y Abrams M.J. (2012). The role of seasonal grass pollen on childhood asthma emergency department presentations. Clin. Exp. Allergy 42, pp. 799-805. DOI: http://dx.doi.org/10. $1111 / \mathrm{j} .1365-2222.2012 .03995 . x$

Eyerich K., Huss-Marp J., Darsow U., Wollenberg A., Foerster S., Ring J., Behrendt H. y Traidl-Hoffmann C. (2008). Pollen grains induce a rapid and biphasic eczematous immune response in atopic eczema patients. Int. Arch. Allergy Immunol. 145: pp. 213-223. DOI: http://dx.doi.org/ $10.1159 / 000109290$.

Fernández F. y Rasilla D.F. (2001). Secular Variations of the Synoptic Circulation over the Iberian Peninsula. In: Brunet MB y Bonillo DL (eds) Detecting and Modelling Regional Climate Change. Springer, Berlin, Heidelberg: pp. 229-238. DOI: http://dx.doi.org/10.1007/978-3-66204313-4_20

Frei T. y Gassner E. (2008). Climate change and its impact on birch pollen quantities and the start of the pollen season an example from Switzerland for the period 1969-2006. Int. J. Biometeorol. 52: pp. 667-674. DOI: http://dx.doi.org/10.1007/s00484-008-0159-2

Frenguelli G., Ghitarrini S. y Tedeschini E. (2014). Climatic change in Mediterranean area and pollen monitoring. Flora Med. 24: pp. 99-107. DOI: http://dx.doi.org/ 24. 10.7320/FlMedit24.099

García M. (1999). Polinosis, una enfermedad en aumento. REA, 5: pp. 13-16.

Greiner A.N., Hellings P.W., Rotiroti G. y Scadding G.K. (2011). Allergic rhinitis. The Lancet 378: pp. 2112-2122. DOI: http://dx.doi.org/10.1016/S0140-6736(11)60130-X

Grundström M., Dahl Å., Ou T., Chen D. y Pleijel H. (2017). The relationship between birch pollen, air pollution and weather types and their effect on antihistamine purchase in two Swedish cities. Aerobiologia, 33 (4). pp. 457-471. DOI: http://dx.doi.org/10.1007/s10453-017-9478-2

Guilbert A., Simons K., Hoebeke L., Packeu A., Hendrickx M., Cremer K., Buyl R., Coomans D. y van Nieuwenhuyse A. (2016). Short-term effect of pollen and spore exposure on allergy morbidity in the Brussels-Capital Region. EcoHealth. 13: pp. 303-315. DOI: http://dx.doi.org/10.1007/ s10393-016-1124-x

Hebbern C.M. y Cakmak, C. (2015). Synoptic weather types and aeroallergens modify the effect of air pollution on hospitalizations for asthma in Canadian cities. Environ. Pollut., 204: pp. 9-16. DOI: http://dx.doi.org/10.1016/j.envpol.2015.04.010

Izquierdo R., Alarcón M., Mazón J., Pino D., De Linares C., Aguinagalde X. y Belmonte J. (2017). Are the Pyrenees a barrier for the transport of birch (Betula) pollen from Central Europe to the Iberian Peninsula? Science of the Total Environment 575: pp. 1183-1196. DOI: http://dx.doi.org/ 10.1016/j.scitotenv.2016.09.192 
Izquierdo R., Belmonte J., Avila A., Alarcón M., Cuevas E. y Alonso-Pérez S. (2011). Source areas and long-range transport of pollen from continental land to Tenerife (Canary Islands). Int J Biometeorol (2011) 55: pp. 67-85. DOI: http://dx.doi.org/10.1007/s00484-010-0309-1

Laiidi K. (2001). Predicting days of high allergenic risk during Betula pollination using weather types. Int. J. Biometeorol. 45: pp. 124-132. DOI: http://dx.doi.org/10.1007/s004840100096

Makra L., Juhász M., Mika J., Bartzokas A., Béczi R. y Sümeghy Z. (2006). An objective classification system of air mass types for Szeged, Hungary, with special attention to plant pollen levels. Int. J. of Biometeorol, 50(6), pp. 403-421. DOI: http://dx.doi.org/10.1007/s00484-006-0026-y

Motreff Y., Golliot F., Calleja M., Le Pape A., Fuhrman C., Farrera I. y Plaisant I. (2014). Short-term effect of pollen exposure on drug consumption for allergic rhinitis and conjunctivitis. Aerobiologia. 2014; 30: pp. 35-44. DOI: http://dx.doi.org/10.1007/s10453-013-9307-1

Paschalidou A., Psistaki K., Charalampopoulos A., Vokou D., Kassomenos P. y Damialis A. (2020). Identifying patterns of airborne pollen distribution using a synoptic climatology approach. Science of The Total Environment. 714. 136625. DOI: http://dx.doi.org/10.1016/j.scitotenv. 2020.136625

Pérez-Badia R., Rapp A., Vaquero C. y Fernández-González F. (2011). Aerobiological study in eastcentral Iberian Peninsula: pollen diversity and dynamics for major taxa. Ann. Agric. Environ. Med. 18: pp. 99-111.

Ribeiro H., Cunha M. y Abreu I. (2003). Airborne pollen concentration in the region of Braga, Portugal, and its relationship with meteorological parameters. Aerobiologia 19: pp. 21-27. DOI: http:// dx.doi.org/10.1023/A:1022620431167

Rojo J., Picornell A. y Oteros J. (2019). AeRobiology: The computational tool for biological data in the air. Methods in Ecology and Evolution. DOI: http://dx.doi.org/10. 10.1111/2041-210 X.13203.

Subiza, J. (2003): Gramíneas: Aerobiología y polinosis en España. Allergol Immunol Clin, 18(3), pp. 7-11.

Todkill D., Gonzalez F., Morbey R., Charlett A., Hajat S., Kovats S., Osborne N., McInnes R., Vardoulakis S., Exley K., Edeghere O., Smith G. y Elliot A. (2020). Environmental factors associated with general practitioner consultations for allergic rhinitis in London, England: a retrospective time series analysis. BMJ Open. DOI: http://dx.doi.org/10. e036724. 10.1136/bmjopen-2019036724 .

Tormo-Molina R., Gonzalo-Garijo M.A., Silva-Palacios I. y Muñoz-Rodríguez A.F. (2010). General trends in airborne pollen production and pollination periods at a Mediterranean site (Badajoz, Southwest Spain). J. Investig. Allergol. Clin. Immunol. 20 (7), pp. 567-574.

Weber R.W. (2003). Meteorologic variables in aerobiology. Immunol. Allergy Clin. N. Am. 23, pp. 411-422.

Werfel T., Heratizadeh A., Niebuhr M., Kapp A., Roesner L.M., Karch A., Erpeck V.J., Lösche C., Jung T., Krug, N., Badorrek P. y Hohlfeld J.M. (2015). Exacerbation of atopic dermatitis on grass pollen exposure in an environmental challenge chamber. J. Allergy Clin. Immunol. 136: pp. 96-103. DOI: http://dx.doi.org/10.1016/j.jaci.2015.04.015

Wilks DS (2019). Statistical methods in the atmospheric sciences. New York: Elsevier. 
